\title{
Exploiting tumour addiction with a serine and glycine-free diet
}

\author{
Ivano Amelio*, ${ }^{*}$ Gerry Melino ${ }^{1,2}$ and Christian Frezza ${ }^{3}$
}

Cell Death and Differentiation (2017) 24, 1311-1313; doi:10.1038/cdd.2017.83; published online 2 June 2017

Understanding cancer metabolism is key to revealing the Achilles' heel of cancer cells and providing novel therapeutic interventions for patients. While the rerouting of metabolic pathways during development ${ }^{1}$ or cancer transformation and progression $^{2-4}$ has been extensively characterised, the exact dynamics of these events, their distribution and frequency in different tumour types, and their correlation with genetic background remain largely unknown. In a recent article published in Nature, Karen Vousden's team assesses the effect of serine and glycine (SG) dietary restriction in autochthonous mouse tumour models driven by different oncogenes, ${ }^{5}$ leading to potential areas of therapeutic intervention.

The non-essential amino acids serine and glycine are now considered essential metabolites for some types of cancers. ${ }^{3,6}$ Serine can be imported into the cell through different transporters or can be produced by diversion of glycolytic glucose in the serine biosynthetic pathway (SSP). ${ }^{7}$ Exogenous or de novo-synthetised serine is converted to glycine, and by stimulating one-carbon metabolism, provides a source of nucleotides, ATP, methylation reactions, and antioxidant defences, such as glutathione and NAPDH. ${ }^{8}$ Cancer cells can react to $S G$ deprivation by triggering SSP, suppressing aerobic glycolysis, and increasing the flux to the tricarboxylic acid cycle. This rearrangement of metabolism supports adaptation to oxidative stress initiated by impaired onecarbon metabolism, and allows cells to survive under these adverse conditions. Notably, the response to serine starvation is regulated by $\mathrm{p} 53$ and, in consequence, $\mathrm{p} 53^{-/-}$cells fail to respond to SG depletion, showing severe impairment of proliferation and cell viability associated with unbalanced redox defence. ${ }^{9}$ Together, these lines of research indicate that SG metabolism can be a potential target for cancer therapy.

As an alternative to selectively targeting the enzymes responsible for SG synthesis, Maddocks et al. ${ }^{5}$ reasoned that SG deprivation could be a powerful strategy to affect this pathway. In their recent study, they analysed the response to an SG-free diet in different genetically modified animal models of lymphoma $(E \mu-M y c)$, intestinal tumours $\left(A p c^{M i n /+}\right)$, and pancreatic cancer (Pdx1-cre; KRas ${ }^{G 12 D /+} ; \operatorname{trp53}^{+/-}$and Pdx1-

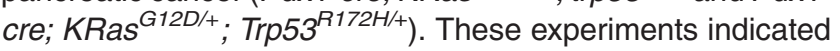
that SG starvation effectively prolongs survival of $E \mu-M y c$ and $A p c^{\mathrm{Min} /+}$ mice, which carry pre-malignant lesions since shortly after birth. The authors also tested the impact of this restricted diet on established malignant tumours. They developed xenograft/allograft models by subcutaneous injection of human colorectal carcinoma (HCT-116) or mouse E $\mu-M y c$ tumour cells and transferred the animals to an SG-depleted experimental diet after the tumours were established. SG restriction reduced tumour volume after relatively short periods of treatment ( 6 days of SG-free diet in E $\mu$-Myc cellsderived tumours) and this was associated with increased cell death in the tumour core.

SG deprivation was also shown to increase mitochondrial metabolism. ${ }^{10}$ Therefore, the authors tested the effects of SG dietary restriction in combination with biguanides and inhibitors of complex I of the electron transport chain. The results highlighted a complex response that suggested that biguanides and SG deprivation might synergise in restricting tumour growth when the combination effectively prevents oxidative defence. Phenoformin indeed reduced tumour growth in $E \mu-M y c$ mice. However, due to its high toxicity, the use of the more tolerable analogue metformin was required to complete the study. Unexpectedly, in SG-deprived $\mathrm{Apc}^{\mathrm{Min} /+}$ mice, pharmacological doses of metformin promoted tumour growth rather than improving the anti-tumour effect. Remarkably, lowand high-dose metformin correlated with increased or decreased reactive oxygen species (ROS) levels, respectively, indicating that the ability of metformin to synergise with SG depletion correlates with its anti- or pro-oxidant effects. To formally prove that increased ROS levels enhance anti-tumour effects of the SG-free diet, the authors crossed E $\mu-M y c$ mice with mice deficient for Tigar, a fructose-2,6-bisphosphatase, which limits glycolysis and favours pentose phosphate pathways, thus limiting ROS levels ${ }^{11,12}$ (Figure 1). The combination of Tigar deficiency and SG deprivation significantly increased overall survival with an additive effect. As a common mechanism of action of routine chemotherapeutic drugs is to attack the cancer cells with high levels of ROS, it is now a priority to test whether an SG-free diet improves efficacy of standard anticancer therapies.

The authors then tested the effects of SG deprivation on a model of pancreatic cancer driven by K-Ras mutation. Surprisingly, the authors found that SG restriction did not affect tumour growth and survival in two different models of Kras-driven pancreatic tumours (Pdx1-cre; KRas ${ }^{G 12 D /+}$; trp53 $^{+/-}$and Pdx1-cre; KRas ${ }^{G 12 D /+}$; Trp53 $\left.{ }^{R 172 H /+}\right)$. Further

${ }^{1}$ MRC Toxicology Unit, Leicester, UK; ${ }^{2}$ Department Experimental Medicine \& Surgery, University Rome Tor Vergata, Rome, Italy and ${ }^{3} \mathrm{MRC}$ Cancer Unit, Cambridge, UK ${ }^{*}$ Corresponding author: I Amelio, MRC Toxicology Unit, Hodgkin Building Lancaster Road, Leicester LE1 9HN, UK. Tel: +44-116-3-252 5564; +44(0)116 252 5599; E-mail: ia119@le.ac.uk 


\section{Standard diet}

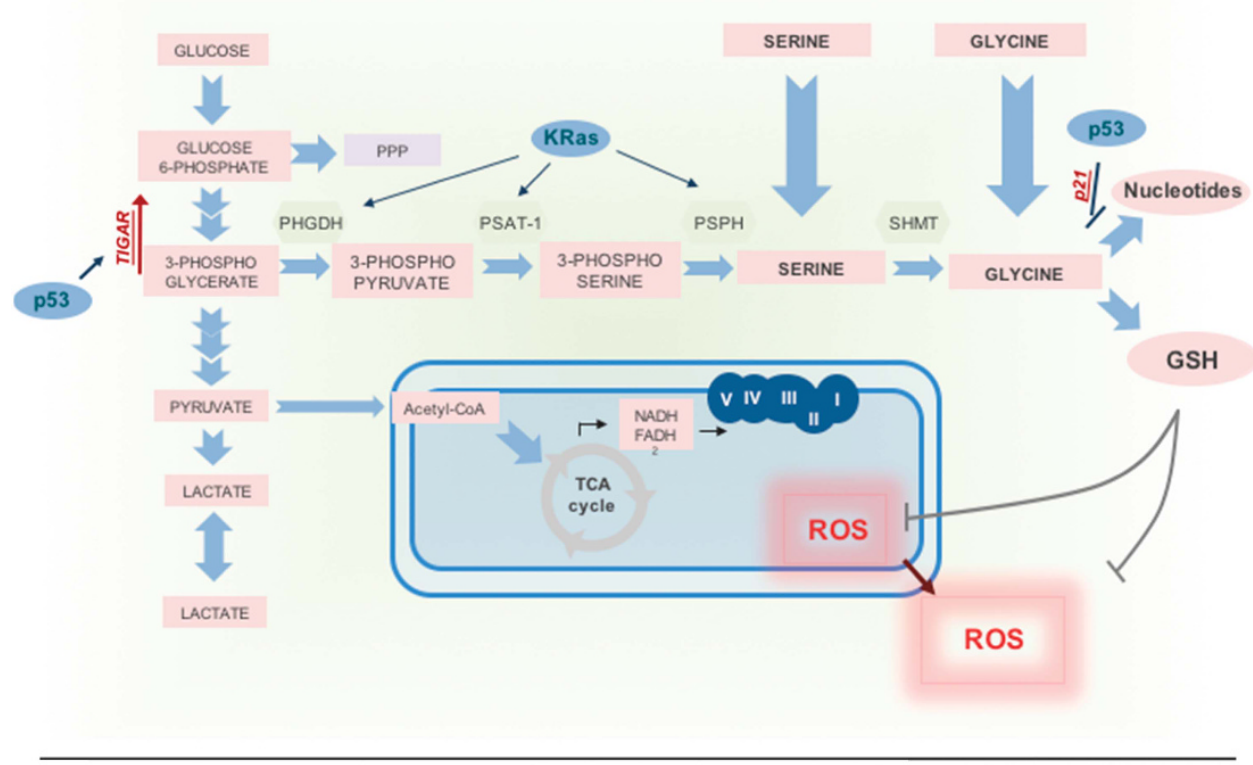

Serine and Glycine free-diet

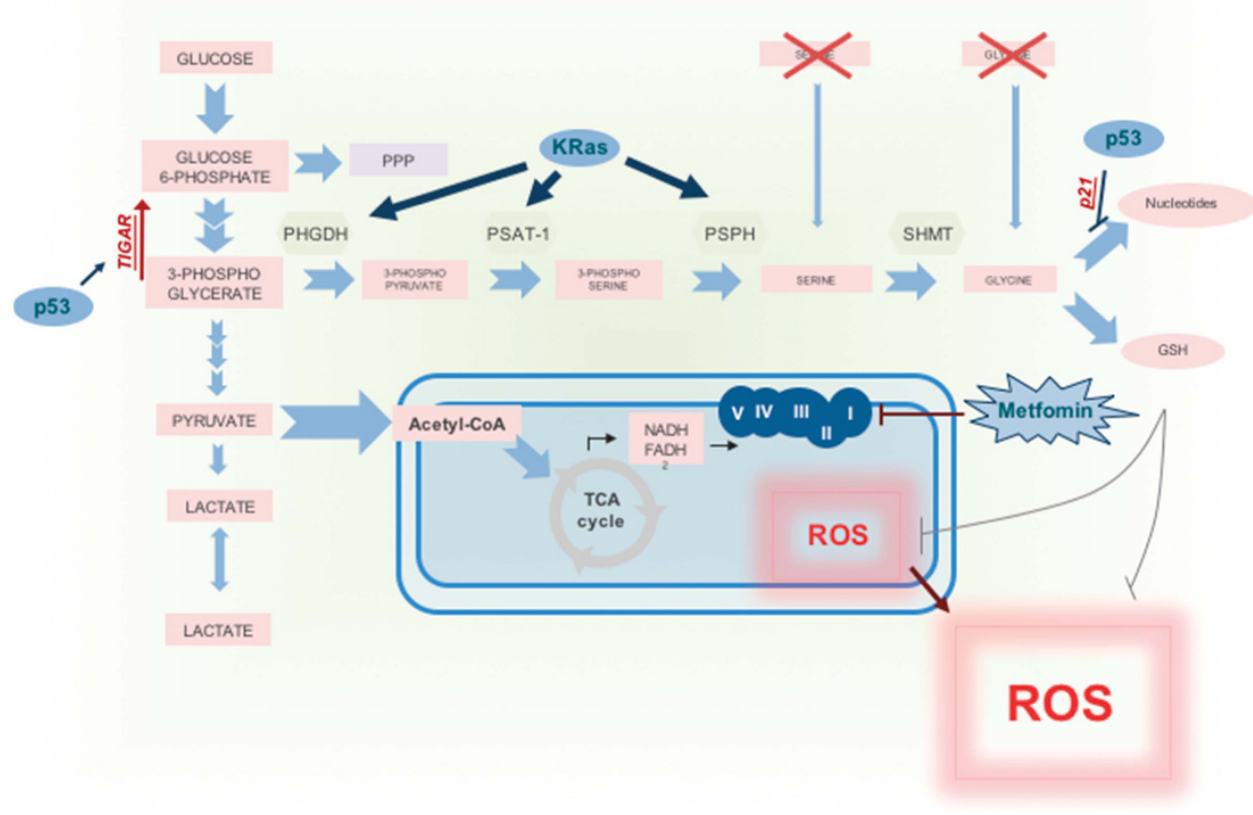

Figure 1 The serine synthesis pathway (SSP) diverges from glycolysis utilising the intermediate 3P-glycerate, which is converted by PHGDH, PSAT-1, and PSPH into serine and then glycine. Removal of exogenous serine and glycine causes activation of SSP, however Vousden's study demonstrates that tumours, such as E $\mu$-Myc-and Apc Min/ + -driven lymphomas and intestinal lesions can be susceptible to serine and glycine (SG) deprivation. Concurrent SG dietary restriction and biguanide administration might improve anti-tumour effects when associated with a reduction in antioxidant defence. On contrary, Kras-driven pancreatic tumours appear resistant to SG-free diet as observed from the ability of Kras to promote expression of PHGDH, PSAT-1, and PSPH and thus boost activity of SSP. This figure is re-adapted from Amelio and Melino ${ }^{19}$

analysis showed that these Kras-driven tumour models rely heavily on SSP for SG supply, when compared to intestinal $A p c^{\mathrm{Min} /+}$ cancers. Analysis of intravenously injected ${ }^{13} \mathrm{C}-{ }^{15} \mathrm{~N}-$ labelled serine in mice revealed that pancreatic tumours take up serine and glycine at a similar level to healthy pancreas; conversely, serine uptake in $A p c^{\mathrm{Min} /+}$ tumours is significantly increased as compared to normal intestinal tissues.
Accordingly, Kras ${ }^{\mathrm{G} 12 \mathrm{D}}$-expressing cells had high levels of SSP enzymes (PHGDH, PAST1, and PSPH), and depletion of Kras reduced their expression (Figure 1). Expression of Kras $^{\mathrm{G} 12 \mathrm{D}}$ in organoids derived from Vil1-creER;APC ${ }^{f / f l}$ intestinal tumours promoted conversion of glucose to serine and thus restored their in vitro growth upon exogenous SG deprivation. These data are in agreement with a recent study 
by Kottakis et al. ${ }^{13}$ reporting that concurrent Kras mutation and LKB1 depletion in the pancreas drives upregulation of SSP and dependence of these cells on this biosynthetic pathway.

In conclusion, these study data highlight the complexity of SG metabolism in cancer cells and the importance of the mutational landscape of cancer in the response to dietary restriction and therapeutic intervention. Indeed, SG deprivation could be ineffective in tumours that exhibit an active SSP. However, in Kras-driven tumours, the effects of SG restriction could be limited in breast tumours characterised by $\mathrm{PHGDH}$ amplification, ${ }^{14,15}$ but also in lung tumours, where NRF2 was shown to activate SSP. ${ }^{16}$ Another important aspect is that the effects of SG deprivation depends on the redox status of the cell. Although the role of oxidative stress in cancer is currently highly debated, this work shows that the effects of SG deprivation could be potentially compromised by strategies that prevent oxidative stress, such as antioxidants. Therefore, any dietary intervention needs to be fine-tuned to prevent idiosyncratic interactions. This work raises several interesting questions. For instance, it will be interesting to assess whether SG-free diets can prevent tumour formation, in addition to slowing its progression. In this context, it is intriguing to speculate that the anticancer effects of low-protein diets or calorie restriction ${ }^{17}$ are, at least in part, mediated by SG restriction. Finally, it will be important to assess the mechanisms beyond the anticancer effects of SG starvation. An SG-free diet affects the immune system leading to a dysregulation of $\mathrm{T}$ lymphocytes, as recently suggested. ${ }^{18}$ It will be interesting to determine whether this diet, besides exhibiting cell-autonomous effects, can actually modulate the immune system to fight cancer cells.

\section{Conflict of Interest}

The authors declare no conflict of interest.

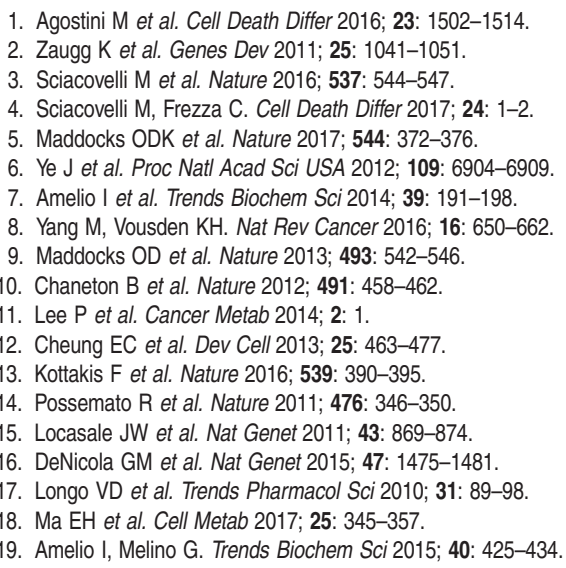

(c) (1) (2) (2) This work is licensed under a Creative Commons Attribution-NonCommercial-ShareAlike 4.0 International License. The images or other third party material in this article are included in the article's Creative Commons license, unless indicated otherwise in the credit line; if the material is not included under the Creative Commons license, users will need to obtain permission from the license holder to reproduce the material. To view a copy of this license, visit http://creativecommons.org/licenses/by-nc-sa/4.0/

C) The Author(s) 2017 